\title{
Multiple Myeloma in a Patient with Castleman's Disease
}

\section{A Sumrall, G Shumaker, S Elkins}

\section{Citation}

A Sumrall, G Shumaker, S Elkins. Multiple Myeloma in a Patient with Castleman's Disease. The Internet Journal of Hematology. 2007 Volume 4 Number 2.

\section{DOI: $\underline{10.5580 / \mathrm{c} 11}$}

\begin{abstract}
Multiple myeloma is characterized by malignant plasma cells in the bone marrow, overproduction of a monoclonal protein, and lytic bony destruction. It is an incurable disease accounting for approximately 20 percent of deaths from hematologic malignancy and 2 percent of deaths from all cancers. 1 It has no established relationships with secondary malignancies; and, the exact cause of the disease is unknown. We will report the first case of multiple myeloma in a patient with multicentric Castleman's disease and explore etiologies of both diseases.
\end{abstract}

\section{INTRODUCTION}

Multiple myeloma (MM) is characterized by malignant plasma cells in the bone marrow, overproduction of a monoclonal protein, and lytic bony destruction. It is an incurable disease accounting for approximately 20 percent of deaths from hematologic malignancy and 2 percent of deaths from all cancers. ${ }_{1}$ It has no established relationships with secondary malignancies; and, the exact cause of the disease is unknown. We will report the first case of multiple myeloma in a patient with multicentric Castleman's disease and explore etiologies of both diseases. 2

\section{CASE REPORT}

A previously healthy 37 year old male presented to clinic in April 2001 complaining of a painless lump in his left inguinal region of 6 months' duration. Physical exam revealed a soft, nonmatted $3 \mathrm{~cm}$ mass in the left inguinal canal. History was unremarkable. Laboratory examination revealed a normal $\mathrm{CBC}$ and chemistry panel. Plain films and CT scan of the chest, abdomen and pelvis showed a lytic lesion of the left pubic ramus, measuring $2.6 \mathrm{~cm}$ by $2.7 \mathrm{~cm}$. The surrounding bone appeared to be slightly irregular with an increased radiographic density, as shown below.
Figure 1

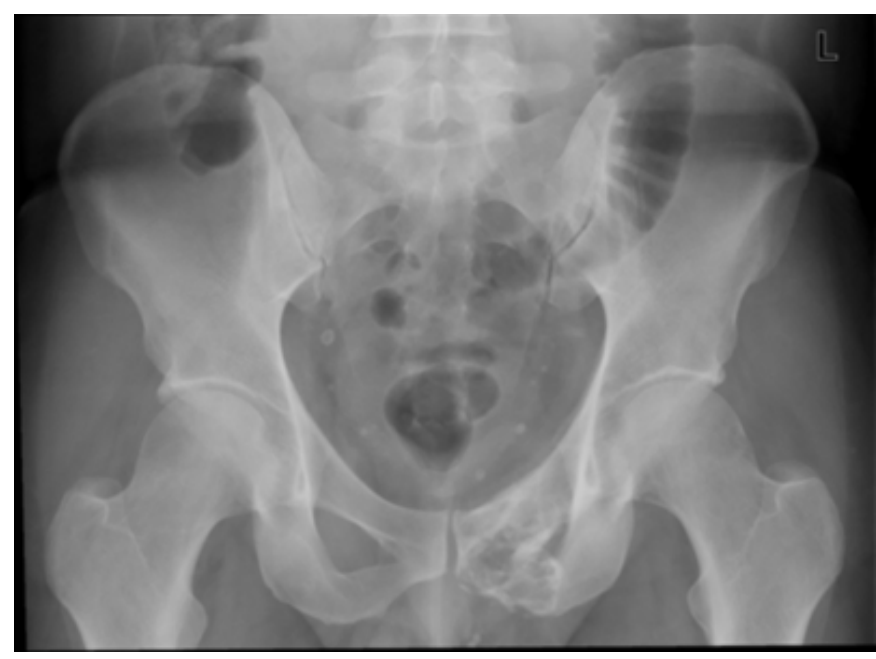

The patient promptly underwent a left inguinal lymph node biopsy which showed angiofollicular lymphoid hyperplasia, which was consistent with Castleman's disease, hyalinevascular type. Flow cytometry failed to identify monoclonal lymphoid cells, ruling out the possibility of malignant lymphoma. Further laboratory investigation by serum protein electrophoresis showed a small monoclonal IgG gammopathy with normal uninvolved immunoglobulins. Bone biopsy of the lytic lesion in the pelvis revealed a monoclonal plasmacytoma for which radiation therapy was initiated. After radiation was complete, the monoclonal gammopathy resolved. A bone marrow aspirate was unremarkable. Skeletal survey in September 2001 showed regular lytic bony destruction of the left pubic bone consistent with monoclonal plasmacytoma. The frontal 
projection of the left knee showed small vague lucencies suspicious for additional bony lesions. The patient was followed every six months by his hematologist. Repeat bone marrow aspiration in October 2001 remained unremarkable. He was doing well until December 2005 when repeat CT scan showed enlargement of the left iliac lesion. Laboratory examination showed recurrence of monoclonal gammopathy. Bone marrow aspirate in January 2006 remained unremarkable.

He was referred to University of Mississippi Medical Center in February 2006 to begin evaluation for bone marrow transplant. After receiving cyclophosphamide for conditioning, the patient had his peripheral stem cells harvested. He began treatment with dexamethasone and thalidomide for multiple myeloma with plans for future transplant.

In July 2007, he underwent autologous peripheral stem cell transplant. He tolerated the procedure well and has remained disease-free.

\section{DISCUSSION}

Castleman's disease (CD) has been associated with malignancies such as Kaposi's sarcoma, lymphoma, and POEMS (polyneuropathy, organomegaly, endocrinopathy, monoclonal gammopathy, and skin changes) syndrome. Etiology of immunological dysfunction associated with CD has remained a mystery, but theories include interleukin- 6 (IL-6) overexpression and human herpesvirus-8 (HHV-8) activity. In animal models, overexpression of IL- 6 by hematopoietic stem cells results in a syndrome comparable to multicentric $\mathrm{CD}$, with peripheral lymphadenopathy, plasma cell infiltration of lymphoid tissues, splenomegaly, anemia, and hypergammaglobulinemia. ${ }_{34}$ Early studies demonstrated reduction of both symptoms and IL-6 levels in patients with $\mathrm{CD}$ who underwent lymph node excision. ${ }_{5,6}$ It has been suggested that blastic B-cells of the germinal centers, follicular dendritic cells, or cells present in the interfollicular regions are responsible for IL-6 production. $5,6,7$

One hypothesis for the origin of multicentric $\mathrm{CD}$, shown in animal and human models, is that HHV-8 expresses viral IL-6, which induces vascular endothelial growth factor (VEGF). ${ }_{4}$ This compound encourages human IL-6 production by endothelial cells. ${ }_{8}$ High levels of IL-6 are frequently seen in patients with CD. While HHV-8 replicates, a viral IL-6 gene (vIL-6) that activates the human IL-6 receptor is expressed. This can cause proliferation of human myeloma cell lines. $9{ }_{90}$ Further investigation has shown that antibodies to human IL-6 block its function, leading to resolution of the systemic symptoms and hypergammaglobulinemia of multicentric CD. ${ }_{11}{ }_{12}$ Although not fully understood at present, it is believed that either viral or human IL-6 is partially responsible for the pathogenesis of $\mathrm{HHV}-8$ positive multicentric CD. ${ }_{13}$ Although these data may address the role of $\mathrm{HHV}-8$ in the pathogenesis of multicentric $\mathrm{CD}$, they do not advance our understanding of the pathogenesis of HHV-8 negative multicentric form. It is likely that these subtypes have a different, unidentified source of immune stimulation.

Although controversial, there is evidence that infection with HHV-8 plays a role in multiple myeloma. ${ }_{14}$ Several small studies have detected HHV-8 in the stromal dendritic cells from bone marrow biopsies of patients with multiple myeloma. HHV-8 was demonstrated in the nonmalignant cell population of long-term bone marrow cultures from patients with MM, as well as in fresh bone marrow biopsy specimens. ${ }_{15},{ }_{16}$ Other studies have confirmed the presence of HHV-8 in bone marrow dendritic cells, but many researchers have not been able to identify HHV-8 in either bone marrow or peripheral blood samples from patients with MM.

$17,18,19,20,21,22,23,24$

Less controversial is the relationship between MM and IL-6. IL-6 is made in bone marrow stromal cells and malignant cells. Production is upregulated by cytokines and CD40. 25 Most MM cell lines and fresh bone marrow MM cells express IL-6 receptors. Binding of IL-6 yields a cascade of effects which activates signal transduction pathways which mediate MM cell growth, survival, and drug resistance. ${ }_{25,26,27}$ Preliminary studies reveal that IL-6 blockade is possible in vivo in patients with MM. ${ }_{28}$

These studies form the basis for our theorized relationship between $\mathrm{MM}$ and multicentric $\mathrm{CD}$. We propose that the two conditions are linked through mechanisms involving HHV-8 and/or IL-6 which could lead to future treatment options. 28 Already ongoing is an investigational study using chimeric anti IL-6 in patients with MM or CD. Development of a human IL-6 antibody could also prove to be helpful in these two diseases.

\section{References}

1. Ries, LAG, Eisner, MP, Kosary, CL, et al. SEER Cancer Statistics Review, 1975-2001. Bethesda, MD, National Cancer Institute, 2004.

2. Hallek, M, Bergsagel, PL, Anderson,KC. Multiple myeloma: Increasing evidence for a multistep transformation 
process. Blood 1998; 91:3.

3. Brandt, SJ, Bodine, DM, Dunbar, CE, Nienhuis, AW. Dysregulated interleukin 6 expression produces a syndrome resembling CD in mice. J Clin Invest 1990; 86:592.

4. Aoki, Y, Jaffe, ES, Chang, Y, et al. Angiogenesis and hematopoiesis induced by Kaposi's sarcoma-associated herpesvirus-encoded interleukin-6. Blood 1999; 93:4034. 5. Yoshizaki, K, Matsuda, T, Nishimoto, N, et al. Pathogenic significance of interleukin-6 (IL-6/BSF-2) in CD. Blood 1989; 74:1360.

6. Leger-Ravet, MB, Peuchmaur, M, Devergne, O, et al. Interleukin-6 gene expression in CD. Blood 1991; 78:2923.

7. Frizzera, G. Atypical lymphoproliferative disorders. In Knowles DM (Ed), Neoplastic Hematopathology,(p. 595), Lippincott, Williams and Wilkins, Philadelphia 2001. 8. Du, MQ, Liu, H, Diss, TC, et al. Kaposi sarcomaassociated herpesvirus infects monotypic (IgMlambda) but polyclonal naive B cells in Castleman disease and associated lymphoproliferative disorders. Blood 2001; 97:2130. 9. Burger, R, Neipel, F, Fleckenstein, B, et al. Human herpesvirus type 8 interleukin- 6 homologue is functionally active on human myeloma cells. Blood 1998; 91:1858. 10. Cannon, JS, Nicholas, J, Orenstein, JM, et al.

Heterogeneity of viral IL-6 expression in HHV-8-associated diseases. J Infect Dis 1999; 180:824.

11. Beck, JT, Hsu, SM, Wijdenes, J, et al. Alleviation of systemic manifestations of CD by monoclonal antiinterleukin-6 antibody. N Engl J Med 1994; 330:602. 12. Foussat, A, Fior, R, Girard, T, et al. Involvement of human interleukin-6 in systemic manifestations of human herpesvirus type 8-associated multicentric CD. AIDS 1999; 13:150.

13. Oksenhendler, E, Carcelain, G, Aoki, Y, et al. High levels of human herpesvirus 8 viral load, human interleukin-6, interleukin-10, and $\mathrm{C}$ reactive protein correlate with exacerbation of multicentric castleman disease in HIVinfected patients. Blood 2000; 96:2069.

14. Sjak-Shie, NN, Vescio, RA, Berenson, JR. The role of human herpesvirus- 8 in the pathogenesis of multiple myeloma. Hematol Oncol Clin North Am 1999; 13:1159. 15. Rettig, MB, Ma, HJ, Vescio, RA, et al. Kaposi's sarcoma-associated herpesvirus infection of bone marrow dendritic cells from multiple myeloma patients. Science 1997; 276:1851.

16. Said, JW, Rettig, MR, Heppner, K, et al. Localization of Kaposi's sarcoma-associated herpesvirus in bone marrow biopsy samples from patients with multiple myeloma. Blood 1997; 90:4278.
17. Chauhan, D, Bharti, A, Raje, N, et al. Detection of Kaposi's sarcoma herpesvirus DNA sequences in multiple myeloma bone marrow stromal cells. Blood 1999; 93:1482. 18. Tisdale, JF, Stewart, AK, Dickstein, B, et al. Molecular and serological examination of the relationship of human herpesvirus 8 to multiple myeloma: orf 26 sequences in bone marrow stroma are not restricted to myeloma patients and other regions of the genome are not detected. Blood 1998; 92:2681.

19. Yi, Q, Ekman, M, Anton, D, et al. Blood dendritic cells from myeloma patients are not infected with Kaposi's sarcoma-associated herpesvirus (KSHV/HHV-8). Blood 1998; 92:402.

20. Mitterer, M, Mair, W, Gatti, D, et al. Dendritic cells derived from bone marrow and CD34+ selected blood progenitor cells of myeloma patients, cultured in serum-free media, do not contain the Kaposi sarcoma herpesvirus genome. Br J Haematol 1998; 102:1338.

21. Brander, C, Raje, N, O'Connor, PG, et al. Absence of biologically important Kaposi sarcoma-associated herpesvirus gene products and virus-specific cellular immune responses in multiple myeloma. Blood 2002; 100:698.

22. Corbellino, M, Pizzuto, M, Bestetti, G, et al. Absence of Kaposi's sarcoma-associated herpesvirus DNA sequences in multiple myeloma. Blood 1999; 93:1110.

23. Tedeschi, R, Kvarnung, M, Knekt, P, et al. A prospective seroepidemiological study of human herpesvirus- 8 infection and the risk of multiple myeloma. Br J Cancer 2001; 84:122. 24. Ablashi, DV, Chatlynne, L, Thomas, D, et al. Lack of serologic association of human herpesvirus-8 (KSHV) in patients with monoclonal gammopathy of undetermined significance with and without progression to multiple myeloma. Blood 2000; 96:2304.

25. Gupta, D, Treon, SP, Shima, Y, et al. Adherence of multiple myeloma cells to bone marrow stromal cells upregulates vascular endothelial growth factor secretion: therapeutic applications. Leukemia 2001; 15:1950.

26. Taga, T, Hibi, M, Hirata, Y, et al. Interleukin-6 triggers the association of its receptor with a possible signal transducer, gp130. Cell 1989; 58:573.

27. Hideshima, T, Nakamura, N, Chauhan, D, Anderson, KC. Biologic sequelae of interleukin-6 induced PI3-K/Akt signaling in multiple myeloma. Oncogene 2001; 20:5991. 28. Van Zaanen H, Lokhorst H, Aarden L, et al. Blocking interleukin-6 activity with chimeric anti-IL6 monoclonal antibodies in multiple myeloma: effects on soluble IL6 receptor and soluble gp130. Leuk Lymphoma 1998 Nov;31 (5-6):551-8. 


\section{Author Information}

Ashley Sumrall, MD

Department of Hematology, University of Mississippi Medical Center

\section{Grace Shumaker, MD}

Jackson Oncology Associates

\section{Stephanie Elkins, MD}

Department of Hematology, University of Mississippi Medical Center 